\title{
Data-driven generalized atomic environment prediction for binary and multinary inorganic compounds using the periodic number
}

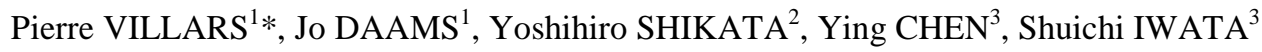 \\ ${ }^{1}$ Material Phases Data System (MPDS), CH-6354 Vitznau, Switzerland \\ ${ }^{2}$ Meijo University, Nagoya, Japan \\ ${ }^{3}$ The University of Tokyo, Tokyo, Japan \\ *Corresponding author. E-mail: villars.mpds@bluewin.ch
}

Received June 9, 2008; accepted June 18, 2008; available on-line September 10, 2008

The atomic environment types AETs (coordination polyhedra) realized in binary and multinary inorganic compounds were analyzed based on a comprehensive set of literature data. The periodic number $P N_{M D}$ (ordering number listing the chemical elements column by column through the periodic system according to

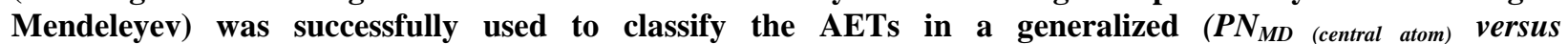
$P N_{M D \text { (coordinating atoms) }}$ ) - AET matrix. Chemical elements with $P N_{M D}>54$ fully control the atomic environment types, regardless of whether they act as central or as coordinating atoms. A generalized AET stability map, using as coordinates $P N_{M D \text { max }}$ vs. $P N_{M D \text { min }} / P N_{M D \text { max }}$, sub-divides the [central atom-coordinating atoms] combinations so that different atomic environment types occur in distinct 'AET class stability domains'. The same matrix (respectively stability map) also shows a clear separation between possible and impossible [central atom-coordinating atoms] combinations. The matrix and stability maps allow, for a chemical element assumed to act as central atom, to predict the most probable AET formed by any coordinating chemical elements, regardless of the stoichiometry and number of chemical elements of the inorganic compound.

Atomic environment types / Coordination polyhedra / Periodic number / Data-driven materials design

\section{Introduction}

\subsection{The aim of this work}

The large amount of experimental structure data collected through the years contains information about correlations that can be used for a semi-empirical approach to materials design. In the ideal case one should be able to relate any kind of "compound property" to elemental-property parameters characteristic of the constituent elements. In a previous study [1], concerning the formation of binary compounds, a clear separation between chemical systems where compounds form and systems where no compounds form was achieved using maps based on combinations of the Mendeleyev Numbers $M D$ of the constituent elements. It was also encouraging that results obtained on binaries could be extended to ternaries and quaternaries. This is an important point because materials design is nowadays more and more focusing on multinaries. But the experimental knowledge available is only substantial for binaries (approximately $70 \%$ of all binary systems have been studied, but less than $5 \%$ of the ternary and less than $0.5 \%$ of the quaternary systems).
After having defined the conditions for the chemical systems where compounds form, it seemed of interest to study closer the structural features of these compounds. For equi-atomic binary $A B$ compounds we were successful in developing an AET map that showed clearly separated 'AET stability domains', using simple expressions of the Mendeleyev Numbers $M D$ of the constituent elements [2]. In the present work we extend this AET map to other binary stoichiometries, as well as to multinary inorganic compounds.

\subsection{Used data}

Having in mind that any classification has a certain degree of ambiguity, we preferred to analyze all individual AETs, rather than to use a prototype classification [3-5] (see under appendix 4.1.). Pearson's Crystal Data CD-ROM, release 2007/8 [6], the most comprehensive crystallographic database for inorganic compounds, contains 150,000 entries (representing about 95,000 different phases) and includes more than 20,000 different prototypes. The 1,000 most populous prototypes represent $80 \%$ of all inorganic compounds. The data in [6] lead to 100 different AETs, 16 of which represent $80 \%$ of all 
the atom sites. The about 290,000 site positions originating from all fully refined structures in [6] lead for the 16 most populous atomic environment types to an average of 14,500 site positions per AET. In conclusion, the approach used here provides a large number of observations per AET and a low number of different AETs.

We use in this work the periodic number $P N_{M D}$ introduced by Villars et al. [7] (see Fig. 1). Differently from the most common representation of the periodic system, $\mathrm{H}$ has been placed on top of the halogen group, and $\mathrm{Be}, \mathrm{Mg}$ on top of group 12 ( $\mathrm{Zn}, \mathrm{Cd}, \mathrm{Hg}$ ). The most common presentation is also called Meier's periodic system, the other one is Mendeleyev's periodic system. The second representation of the Periodic Table is strongly supported by our previous, as well as by the present study.

The Mendeleyev number was introduced by Pettifor in 1984 [8], based on a phenomenological optimization of the separation of binary $A B$ compounds into different prototypes. Basically, the string of Mendeleyev numbers runs through the periodic system group by group. The particular optimization by Pettifor had the effect to place Eu and $\mathrm{Yb}$ just after $\mathrm{Ca}, \mathrm{Pb}$ after $\mathrm{Ga}$, etc.

In the present investigation we used structural information contained in [6]. Prototypes, as well as the atomic environment types AETs are retrievable parameters in [6] and can easily be visualized with the help of its integrated software. Additional information on chemical systems where no compounds are formed was taken from Okamoto [9], from PAULING FILE Binaries Edition CDROM, release 2002 [10], and from ASM Alloy Phase Diagrams Center, onlinerelease 2007/8 [11].

A chemical system is defined as a former (here explained for the binary case) if, at atmospheric pressure, it contains at least one phase that is separated from the terminal solid solutions of the constituent elements by a two-phase region. A system that does not fulfill this criterion is called a non-former. Nonformer systems are characterized by enthalpies of formation that are either positive or close to zero, in agreement with the theory of Miedema [12]. Twophase mixtures of the constituent elements (simple eutectic, simple peritectic and complete insolubility), as well as complete solid solutions are non-formers. Systems with terminal or complete solid solutions where ordered phase areas are observed are also considered as non-formers, because the enthalpy of formation associated with the ordering of the atoms at the positions of the elemental crystal structure is very close to zero.

\section{Results}

\subsection{Observed Atomic Environment Types AETs}

The coordination of each crystallographic point-set in roughly 65,000 refined structures of inorganic compounds from [6] was examined. In total, the AETs of over 290,000 atom sites (point-sets) were considered. In this study the following information was taken into consideration: AET, the nature of the central atom, hereafter referred to as $A$, and the nature of the coordinating atoms $\left(B_{x} C_{y} D_{z}\right)$, hereafter referred to as $B$, e.g. octahedron, $\mathrm{Na}, \mathrm{F}_{2} \mathrm{O}_{4}$; tetrahedron, $\mathrm{Na}$, $\mathrm{As}_{4}$.

The result shows that 100 different AETs are realized in the 65,000 inorganic compounds, but AETs such as non-collinear, non-coplanar triangle, tetrahedron, octahedron, pentagonal pyramid, icosahedron, cuboctahedron, and rhombic dodecahedron largely predominate. The 16 most populous AETs, representing 234,562 sites (80\%), are shown in Fig. 2 and their counts are given in Table 1. Fig. 1 shows the 'Zintl line' considered in this work, and gives the counts per chemical element (in total more than 565'000 sites). Sites with mixed occupation, disorder such as site spitting or partial vacancies were not taken into consideration. Incorrect or incomplete structure determinations, which often generate 'strange asymmetrical' AETs, are excluded from our considerations.

The following observations have been made:

- The maximum number of different chemical elements among the coordinating atoms $B$ within an AET is 3 .

- The maximum number of coordinating atoms observed in AETs where the central atom is an element located on the right-hand side of the 'Zintl line' is 9 .

- The minimum number of coordinating atoms observed in AETs where the central atom is an element located on the left-hand side of the 'Zintl line' is 12 .

- The distribution of different chemical elements among the coordinating atoms $B$ within the AET is in general limited to the highest symmetrical distribution, although, in principle, there exist many other possibilities to distribute them.

- By far the highest occurrence counts of [central atom-coordinating atoms] combinations are realized when chemical elements located to the right of the 'Zintl line' act as central atom. The top-10 with decreasing counts are $\mathrm{O}, \mathrm{H}, \mathrm{S}, \mathrm{F}, \mathrm{C}$, $\mathrm{Si}, \mathrm{P}, \mathrm{N}$, and $\mathrm{Cl}$, as well as the $\mathrm{s}^{1}$-element $\mathrm{Na}$ (see Fig. 1).

- The counts for central atoms having the same group number $G N$ clearly decrease with increasing main quantum number $Q N$, regardless of the kind of the coordinating atoms. Most data are available for central atoms with $Q N 2$ and 3.

- AETs with even coordination numbers $\mathrm{CN}$ are strongly preferred. AETs with CN 5, 7, 10, 11, 13, 15 occur only very exceptionally.

- The 16 most populous AETs are highly symmetrical, and realize a 'spherical' distribution of the coordinating atoms within the AET. 


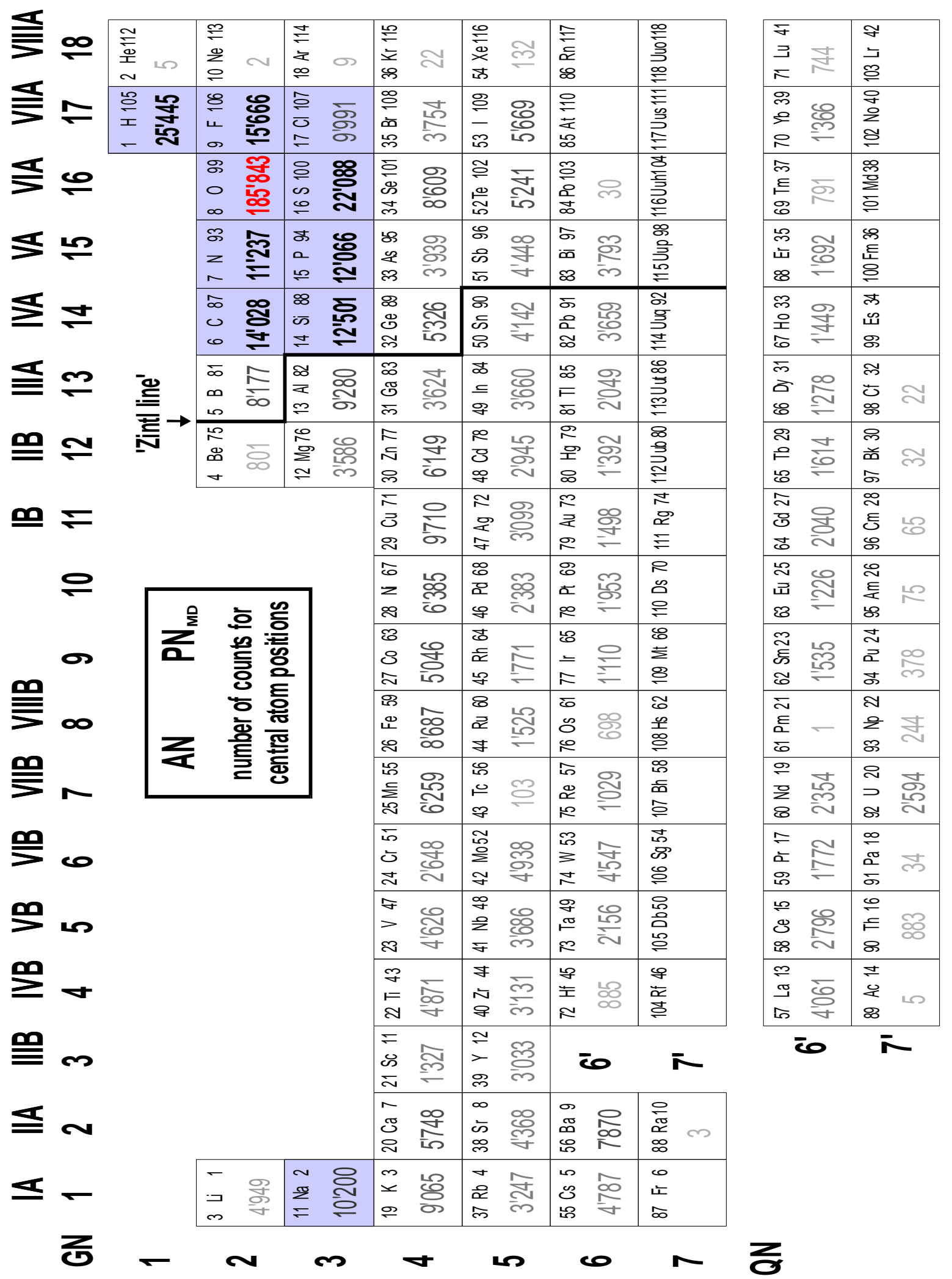

Fig. 1 Mendeleyev type Periodic Table including the atomic numbers $A N$ and periodic numbers $P N_{M D}$, as well as the 'Zintl line' considered in this work. The occurrence count (number of times the chemical element occurs as central atom) for the data set used here is indicated for each element. The highlighted elements have an outstanding high occurrence frequency as central atom. 


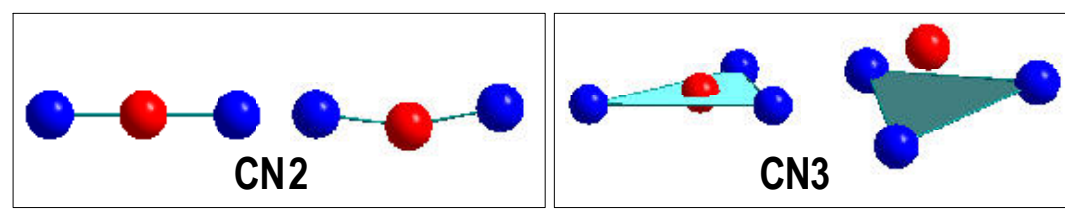

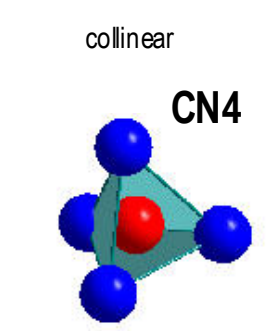

tetrahedron

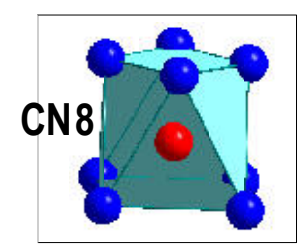

square antiprism

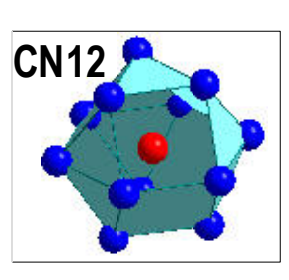

anticuboctahedron coplanar triangle

non-coplanar triangle

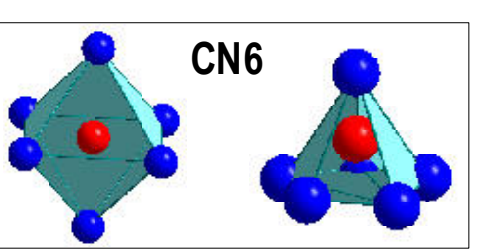

octahedron

pentagonal pyramid

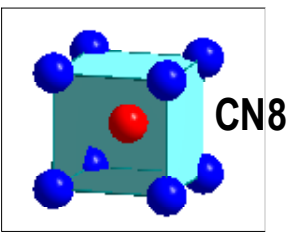

square prism

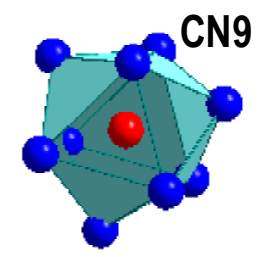

tricapped trigonal prism
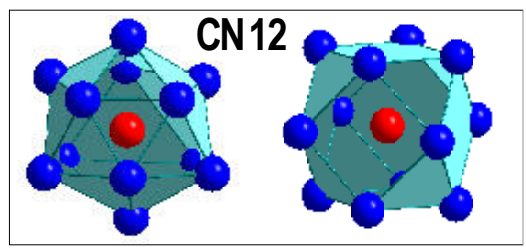

icosahedron

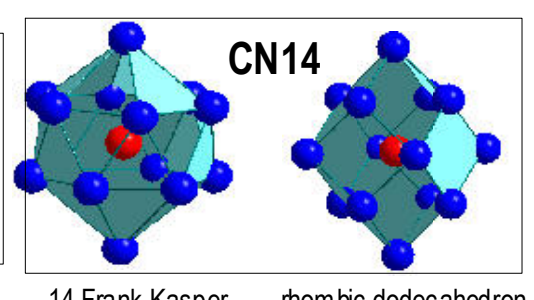

14 Frank-Kasper rhombic dodecahedron

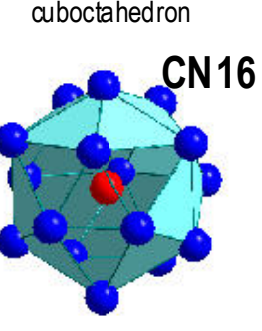

16 Frank-Kasper

Fig. 2 The 16 most populous AETs of about 65,000 refined inorganic compounds published in Pearson's Crystal Data CD-ROM, release 2007/8 [6].

Table 1 The 16 most frequently occurring Atomic Environment Types in Pearson's Crystal Data CD-ROM, release 2007/8 [6] (refined structures).

\begin{tabular}{c|l|l}
\hline CN & Occurrence & Name \\
\hline 2 & 6,377 & collinear \\
& 47,299 & non-collinear \\
3 & 3,923 & coplanar triangle \\
& 22,104 & non-coplanar triangle \\
4 & 39,314 & tetrahedron \\
6 & 44,580 & octahedron \\
& 11,181 & pentagonal pyramid \\
8 & 5,006 & square prism (cube) \\
9 & 3,747 & square antiprism \\
12 & 8,550 & tricapped trigonal prism \\
& 9,331 & icosahedron \\
14 & 14,498 & cuboctahedron \\
16 & 2,875 & anticuboctahedron \\
& 3,517 & 14-vertex Frank-Kasper \\
& 8,300 & rhombic dodecahedron \\
\hline
\end{tabular}


2.2. Generalized Atomic Environment Type Matrix: $P N_{M D}(A)$ versus $P N_{M D}(B)-A E T$ Matrix

Fig. 3 shows the distribution of the AETs observed for the 234,562 sites considered, with the additional restraint that they have an occurrence count larger than 3 per [central atom-coordinating atoms] combination. This additional criterion was necessary to exclude special, as well as exotic cases. Through the introduction of this additional criterion, $2.5 \%$ of the 234,562 sites were excluded.

In addition, information about binary systems where equi-atomic compounds do not form, which coincide with the not observed [central atomcoordinating atoms] combinations (binary nonformers), is included [2]. This leads to the idealized square- or rectangular-shaped non-former domains used in this work, the following 30 binary systems represent violations having stable equi-atomic compounds: Ba-Na, Bi-In, Bi-Mn, Bi-Pt, Bi-Rh, Br-H, Br-I, C-Si, Cd-Hg, Cd-Mg, Cl-H, Cl-I, F-H, Fe-Tc, $\mathrm{Ga}-\mathrm{Mg}, \mathrm{H}-\mathrm{I}, \mathrm{Hg}-\mathrm{In}, \mathrm{Hg}-\mathrm{Mg}, \mathrm{Hg}-\mathrm{Mn}$, In-Mg, In-Ni, In$\mathrm{Pd}$, In-Rh, Ir-Pb, Mg-Tl, Mn-Zn, Pb-Pd, Pb-Pt, Pb-Rh, and Pt-Tl.

The element occupying the center of the AET, $A$, is given on the $y$-axis and the elements acting as coordinating atoms, $B$, on the $x$-axis. It should be noted that a chemical element acting as $A$ can also be part of the coordinating atoms $B$. For cases where the coordinating atoms consist not only of one kind of element, but also of other elements, the same AET has been indicated at several positions in the AET matrix, e.g. octahedron $(\mathrm{CN}=6)$ for $y=\mathrm{Na}, x=\mathrm{F}$, as well as for $y=\mathrm{Na}, x=\mathrm{O}$ for the example given under 2.1. On both axes the chemical elements are ordered according to the periodic number $P N_{M D}$ defined above.

Each element combination is present twice in the AET matrix, once the considered element is acting as central atom $A$ (rows), and once it is acting as coordinating atom $B$ (columns). The [central atomcoordinating atoms] combinations with $P N_{M D}(A)$ larger than $P N_{M D}(B)$ are situated to the left of the diagonal 1/1-118/118, whereas the [central atomcoordinating atoms] combinations with $P N_{M D}(A)$ smaller than $P N_{M D}(B)$ are situated to the right of it. The diagonal in Figs. 3-5 have been left blank due to the fact that for these cases no restrictions seem to exist in context of the possible AETs.

The [central atom-coordinating atoms] combinations that do not occur are located symmetrically with respect to the diagonal 1/1$118 / 118$. The overall picture reveals a strong pseudosymmetry with respect to the diagonal, due to the dominating influence of the valence electron configuration of the central atom $A$ on the choice of AET when $P N_{M D}(A)>P N_{M D}(B)$ (see below). On the contrary, for combinations with $P N_{M D}(A)<P N_{M D}(B)$, AET is mainly determined by the valence electron configuration of the chemical elements acting as coordinating atoms. Chemical elements with $P N_{M D}>$ 54 determine the atomic environment types, regardless of whether they act as central or as coordinating atoms.

The fact that the 'non-existence' of [central atomcoordinating atoms] combinations is consistent with the 'non-existence' of binary equi-atomic compounds demonstrates that if the 'reactivity difference' is too small in the binary case, it is also too small for realizing [central atom-coordinating atoms] combinations within any AET, even including other chemical elements. The domains of not observed [central atom-coordinating atoms] combinations are located along the diagonal in the generalized AET matrix, and their size decreases from $P N_{M D}(A)=$ $P N_{M D}(B)=1$ to $P N_{M D}(A)=P N_{M D}(B)=118$. This is consistent with the behavior of the atomic reactivity $R E_{a}$ as a function of $P N_{M D}$ (see under appendix 4.3.). There exist seven non-former squares along the diagonal 1/1-118/118. Three of them are large, the first one including all elements with $P N_{M D}<55$, i.e. $\mathrm{s}^{1,2}-, \mathrm{f}^{1-14}-$, and $\mathrm{d}^{1-4}$-elements, the second one $\mathrm{d}^{5-9}$ elements and the third one $\mathrm{d}^{10}-$ and $\mathrm{p}^{1,2}$-elements. The four smaller ones contain either $\mathrm{p}^{3}-, \mathrm{p}^{4}-, \mathrm{p}^{5}$, or $\mathrm{p}^{6}$ elements. Significant extensions of the squares are observed for $\mathrm{p}^{1}-, \mathrm{p}^{2}$ - and $\mathrm{p}^{3}$-elements, but only for those with high main quantum number QN (having in mind that the reactivity of the elements decreases with increasing main quantum number $Q N$ for any considered group number $G N$ ).

The observations made above allow a straightforward filling-up of the empty fields in Fig. 3, leading to a 'prediction-completed' generalized AET matrix valid for all potential [central atomcoordinating atoms] combination possibilities, shown in Fig. 4. Several well-defined 'AET class stability domains', which can be simply formulated as ' $P N_{M D}(A)$ range - $P N_{M D}(B)$ range' rectangular domains, are observed within the AET matrix.

In order to obtain the simple separation into distinct 'AET class stability domains' shown in Figs. 3 and 4 it appeared convenient to group several AETs together in AET classes. This is related to the fact that we had to reduce the problem to a simplified situation where the coordinating atoms, which can be at most three different chemical elements, are plotted along one axis, $P N_{M D}(B)$. In the extreme case the same AET has been indicated for three different element combinations, $A-B, \quad A-B$ ' and $A-B$ ". When considerably more data will be available it might be possible to 'separate' these AET class domains into distinct AET domains by taking the additional chemical elements into account in a different way. As a direct consequence of the method used here, for a given central atom $A$, only chemical elements that are located within the same 'AET class stability domain' in the AET matrix can co-act as coordinating atoms $B$. Chemical elements situated outside the 'AET class stability domain' cannot occur within the same atomic environment. In this work we have created 12 different AET classes (see Table 2). The necessity to introduce these AET classes is reinforced by the fact 
P. Villars et al., Data-driven generalized atomic environment prediction for binary and ...
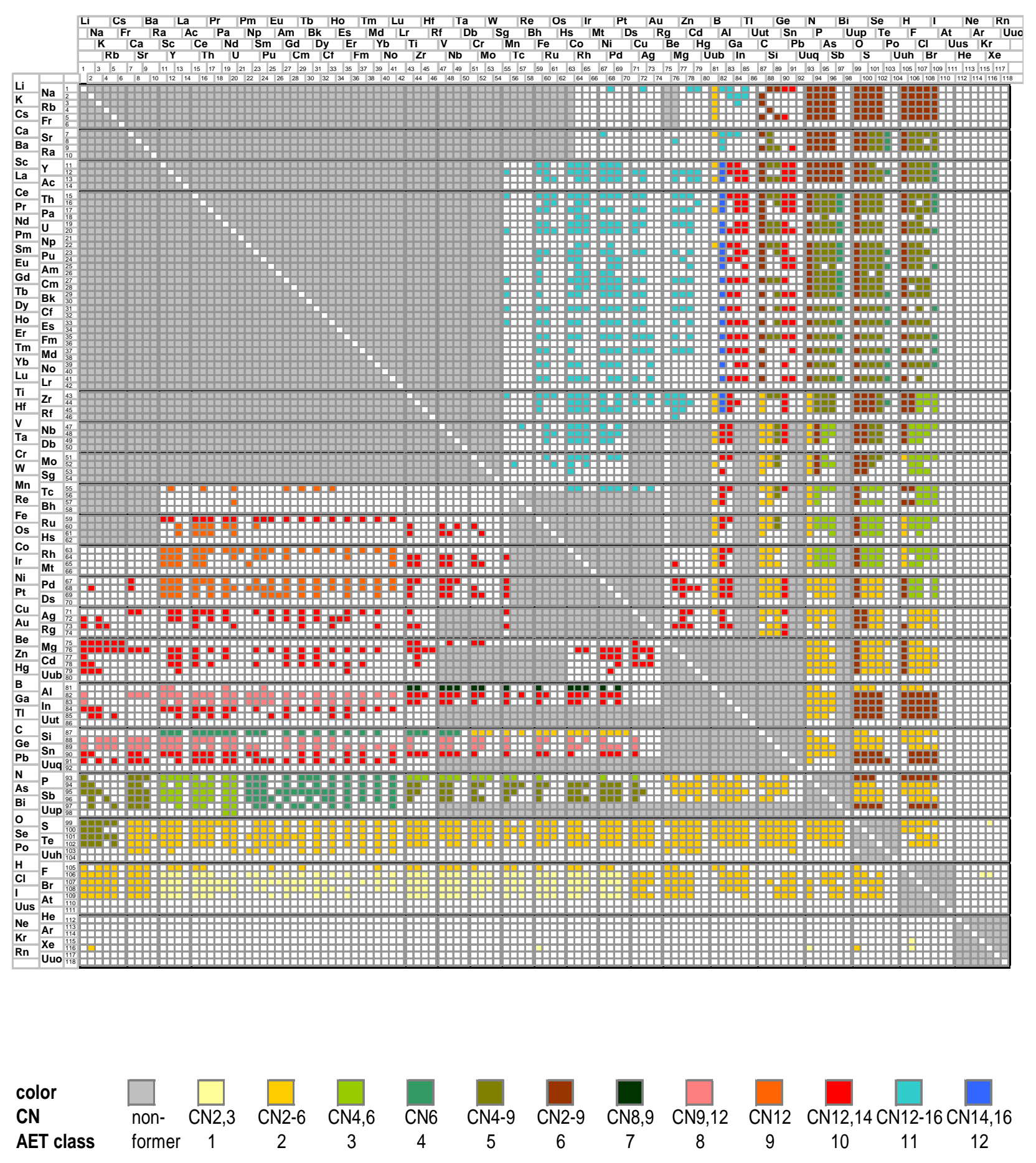

Fig. 3 Generalized AET matrix $P N_{M D}(A)$ vs. $P N_{M D}(B)$, which is independent of the stoichiometry and the number of chemical elements in the compound (based on 65,000 inorganic compounds, which have a [central atom-coordinating atoms] combination - AET occurrence count higher than 3). The element $A$ occupying the center of the AET is given on the $y$-axis and the coordinating elements $B$ on the $x$-axis. For each element $B$ acting as coordinating atom the total $\mathrm{CN}$ is considered. 
P. Villars et al., Data-driven generalized atomic environment prediction for binary and ...



Fig. 4 Generalized AET matrix $P N_{M D}(A)$ vs. $P N_{M D}(B)$, which is independent of the stoichiometry and the number of chemical elements in the compound, prediction-completed for all possible [central atomcoordinating atoms] combinations. The element $A$ occupying the center of the AET is given on the $y$-axis and the coordinating elements $B$ on the $x$-axis. For each element $B$ acting as coordinating atom the total $\mathrm{CN}$ is considered.

that only about $1 / 3$ of all atom sites considered here belong to single- or two-atomic environments types, whereas the remaining $2 / 3$ belong to three- or higheratomic environments types (see under appendix 4.1.) It should be noted that the AET matrix presented here is different from the better-known structure maps where each $A B-, A B_{2^{-}}, A_{2} B_{3^{-}}, A B C$-, etc. compound is characterized by its prototype in separate isostoichiometric $A B-, A B_{2^{-}}, A_{2} B_{3^{-}}, A B C$-, etc. structure maps.

A good separation of all potential [central atomcoordinating atoms] combinations - AETs, including domains where no such combinations are observed, is achieved by this simple generalized AET matrix. No attempt was made to optimize the periodic number $P N_{M D}$ by modifying the order of the rows and columns in the Periodic Table. It is, however, worth noting that when the periodic number $P N_{M D}$ is replaced by any of the other 38 selected elemental-property parameters listed in reference [7] the resulting generalized AET matrixes show a lower degree of local AET class ordering.

From Fig. 5, which shows the filled-up AET matrix in a slightly different presentation, it can be concluded that the AETs are in first priority controlled by the valence electron configurations of the 
Table 2 Atomic Environment Type AET classes used in this work.

\begin{tabular}{|c|c|c|}
\hline $\begin{array}{l}\text { AET } \\
\text { class }\end{array}$ & $\begin{array}{l}\text { Coordination } \\
\text { numbers } \mathrm{CNs}\end{array}$ & Atomic environment types AETs \\
\hline 1 & 2,3 & (non-) collinear, (non-) coplanar triangle \\
\hline 2 & $2-6$ & $\begin{array}{c}\text { (non-) collinear, (non-) coplanar triangle, tetrahedron, octahedron, pentagonal } \\
\text { pyramid }\end{array}$ \\
\hline 3 & 4,6 & tetrahedron, octahedron, pentagonal pyramid \\
\hline 4 & 6 & octahedron, pentagonal pyramid \\
\hline 5 & 4-9 & $\begin{array}{c}\text { tetrahedron, octahedron, pentagonal pyramid, square prism, square antiprism, } \\
\text { tricapped trigonal prism }\end{array}$ \\
\hline 6 & $2-9$ & $\begin{array}{l}\text { (non-) collinear, (non-) coplanar triangle, tetrahedron, octahedron, pentagonal } \\
\text { pyramid, square prism, square antiprism, tricapped trigonal prism }\end{array}$ \\
\hline 7 & 8,9 & square prism, square antiprism, tricapped trigonal prism \\
\hline 8 & 9,12 & tricapped trigonal prism, icosahedron, cuboctahedron, anticuboctahedron \\
\hline 9 & 12 & icosahedron, cuboctahedron, anticuboctahedron \\
\hline 10 & 12,14 & $\begin{array}{c}\text { icosahedron, cuboctahedron, anticuboctahedron, } 14 \text { Frank-Kasper, rhombic } \\
\text { dodecahedron }\end{array}$ \\
\hline 11 & $12-16$ & $\begin{array}{c}\text { icosahedron, cuboctahedron, anticuboctahedron, } 14 \text { Frank-Kasper, rhombic } \\
\text { dodecahedron }\end{array}$ \\
\hline 12 & 14,16 & 14 Frank-Kasper, rhombic dodecahedron, 16 Frank-Kasper \\
\hline
\end{tabular}
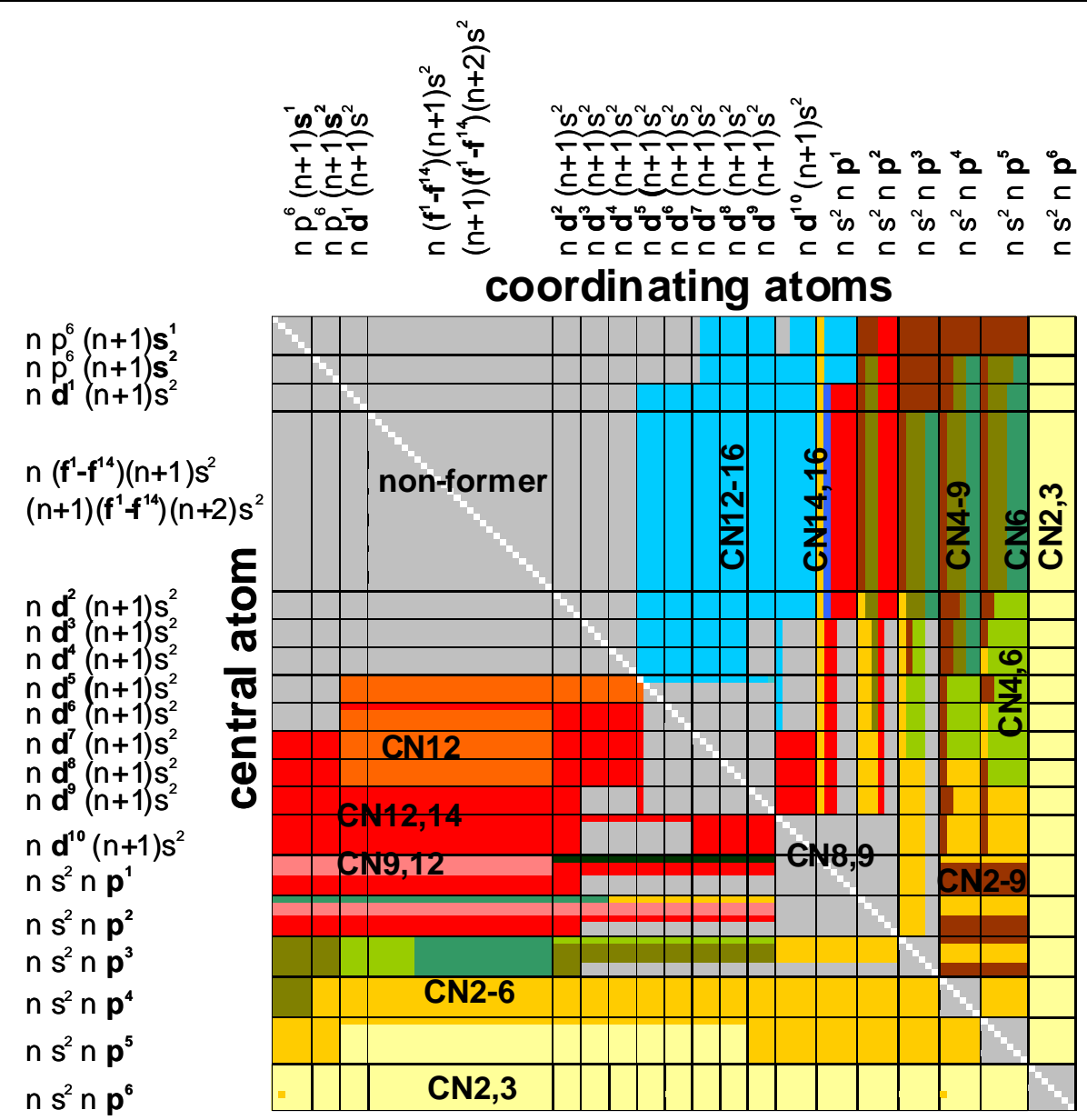

Fig. 5 Schematic Generalized AET map $P N_{M D}(A)$ vs. $P N_{M D}(B)$ highlighting the correlation to the valence electron configuration of the constituent chemical elements. The element $A$ occupying the center of the AET is given on the $y$-axis and the coordinating elements $B$ on the $x$-axis. For each element $B$ acting as coordinating atom the total $\mathrm{CN}$ is considered. 
constituent elements. The sizes of the atoms, often considered as being important in this context, appear to be almost irrelevant. The relative size of the atoms is, however, responsible for the frequent distortions observed within the AETs.

\subsection{Generalized Atomic Environment Type Stability} Map: $P N_{M D} \max$ versus $P N_{M D} \max / P N_{M D \min }-A E T$ Stability Map

The pattern observed in the generalized AET matrix $P N_{M D}(A)$ vs. $P N_{M D}(B)$ motivated us to search systematically for other simple mathematical correlations by combining elemental-property parameters EPs, which could be used as potential axes.

Thus we combined 39 different elemental-property parameters, listed in [7], of the constituent elements $\left(\mathrm{EP}_{\mathrm{A}}\right.$ and $\left.\mathrm{EP}_{\mathrm{B}}\right)$ by applying simple mathematical operators $(+,-, *, /$, min, $\max )$. The following expressions were used, where the elemental-property parameters can be the atomic number $A N$, group number $G N$, atomic size $S Z_{a}$, atomic reactivity $R E_{a}$, first ionization potential, etc.:

$$
\begin{array}{ll}
\text { Sum } & =\mathrm{EP}_{\mathrm{A}}+\mathrm{EP}_{\mathrm{B}} \\
\text { Difference } & =\left|\mathrm{EP}_{\mathrm{A}}-\mathrm{EP}_{\mathrm{B}}\right| \\
\text { Product } & =\mathrm{EP}_{\mathrm{A}} * \mathrm{EP}_{\mathrm{B}} \\
\text { Ratio } & =\mathrm{EP}_{\mathrm{A}} / \mathrm{EP}_{\mathrm{B}}, \text { with } \mathrm{EP}_{\mathrm{A}}<\mathrm{EP}_{\mathrm{B}} \\
\text { Maximum } & =\max \left(\mathrm{EP}_{\mathrm{A}}, \mathrm{EP}_{\mathrm{B}}\right) \\
\text { Minimum } & =\min \left(\mathrm{EP}_{\mathrm{A}}, \mathrm{EP}_{\mathrm{B}}\right)
\end{array}
$$

The data set was divided into two sub-sets:

$P N_{M D \max }(A)$ - AET data set: The chemical element acting as central atom $A$ has higher periodic number than the element acting as coordinating atom $B$, $P N_{M D}(A)>P N_{M D}(B)$. This data set corresponds to the $A-B$ combinations to the left of the $1 / 1-118 / 118$ diagonal in the AET matrix.

$P N_{M D \min }(A)$ - AET data set: The chemical element acting as central atom $A$ has lower periodic number than the element acting as coordinating atom $B$, $P N_{M D}(A)<P N_{M D}(B)$. This data set corresponds to the $A-B$ combinations to the right of the $1 / 1-118 / 118$ diagonal in the AET matrix.

All possible two- and three-dimensional elementalproperty parameter combinations, e.g. sum $=\mathrm{EP}_{\mathrm{A}}+$ $\mathrm{EP}_{\mathrm{B}}$ vs. product $=\mathrm{EP}_{\mathrm{A}} * \mathrm{EP}_{\mathrm{B}}$ vs. $\min \left(\mathrm{EP}_{\mathrm{A}}, \mathrm{EP}_{\mathrm{B}}\right)$, were investigated and the correlations evaluated by the closest-neighbor (domain) method. The best separation into 'AET class domains' was realized for the generalized AET stability map $P N_{M D \max } v s$. $P N_{M D \text { min }} / P N_{M D \text { max }}$ using the two data sets defined above. The result is presented in Fig. 6, which shows a pseudo-mirror plane at $P N_{M D \min }=P N_{M D \max }$. In the upper map the central atom $A$ is $P N_{M D \max }$ in comparison with the $P N_{M D}$ of the coordinating atom $B$, whereas in the lower map the central atom $A$ is $P N_{M D}$ min in comparison with the $P N_{M D}$ of the coordinating atom $B$. Analogically to the AET matrix, in the extreme case the same AET has been indicated for three different element combinations, $A-B, A-B$ ' and $A-B$ ". As a direct consequence this means that, for a given element acting as central atom $A$, only elements located in the same 'AET class stability domain' in the AET stability map are possible within the same atomic environment and chemical elements outside that 'AET class stability domain' cannot occur within the same atomic environment.

The separation of the different AET classes of [central atom-coordinating atoms] combinations into distinct 'AET class stability domains' is satisfactory and several observations can be made. First of all, the fields where no [central atom-coordinating atoms] combinations are observed are clearly separated from those with existing [central atom-coordinating atoms] combinations. The majority of the former correspond to combinations of elements with periodic numbers $P N_{M D}$ lower than $55(\mathrm{Mn})$. The ordering according to the maximum periodic number ( $y$-axis) has a very strong separating effect, also for the different AET classes. The ratio $P N_{M D \text { min }} / P N_{M D \text { max }}$ $(x$-axis) is, however, necessary to achieve a satisfactory separation. For each chemical element acting as central atom the [central atom-coordinating atoms] combinations are equally distributed along the line $0-1-0$, realizing a maximum spread.

From top to bottom in the graphs the chemical elements appear in the order I, Br, Cl, F, H, Po, Te Se, etc., each element being represented by one row. With increasing value of the periodic number $P N_{M D}$ one goes from not observed [central atom - coordinating atoms] combinations to existing [central atomcoordinating atoms] combinations. Among the existing [central atom-coordinating atoms] combinations the coordination number decreases from 16 to 1 . From the definition of $P N_{M D}$ it follows that with increasing periodic number $P N_{M D}$ the group number $G N$ is increasing from 1 to 18 . [Central atomcoordinating atoms] combinations formed by elements with similar periodic numbers are situated close to the vertical line at $x=1$, and correspond dominantly to non-former systems. Even the stronger tendency towards non-formers with increasing $Q N$ for the same $G N$ is clearly seen. [Central atomcoordinating atoms] combinations with the same ratio $P N_{M D \min } / P N_{M D \max }$ ( $x$-axis) can be found along vertical lines. The values $1 / 3,1 / 2$ and $2 / 3$ show the highest distribution density. $P N_{M D}$ up to 104 are included.

Analogous to Fig. 6, Fig. 7 shows the two data sets 'prediction-completed' for all potential [central atomcoordinating atoms] combinations. As for the AET matrix (Fig. 4), with these two sub-graphs it is straight forward to check the most probable AET of any chemical element acting as central atom $A$ with any potential coordinating atoms $B$. This is interesting from a modeling perspective point of view, but also to compare the AET of newly investigated inorganic compounds with the general trend observed for the majority of all known inorganic compounds. 

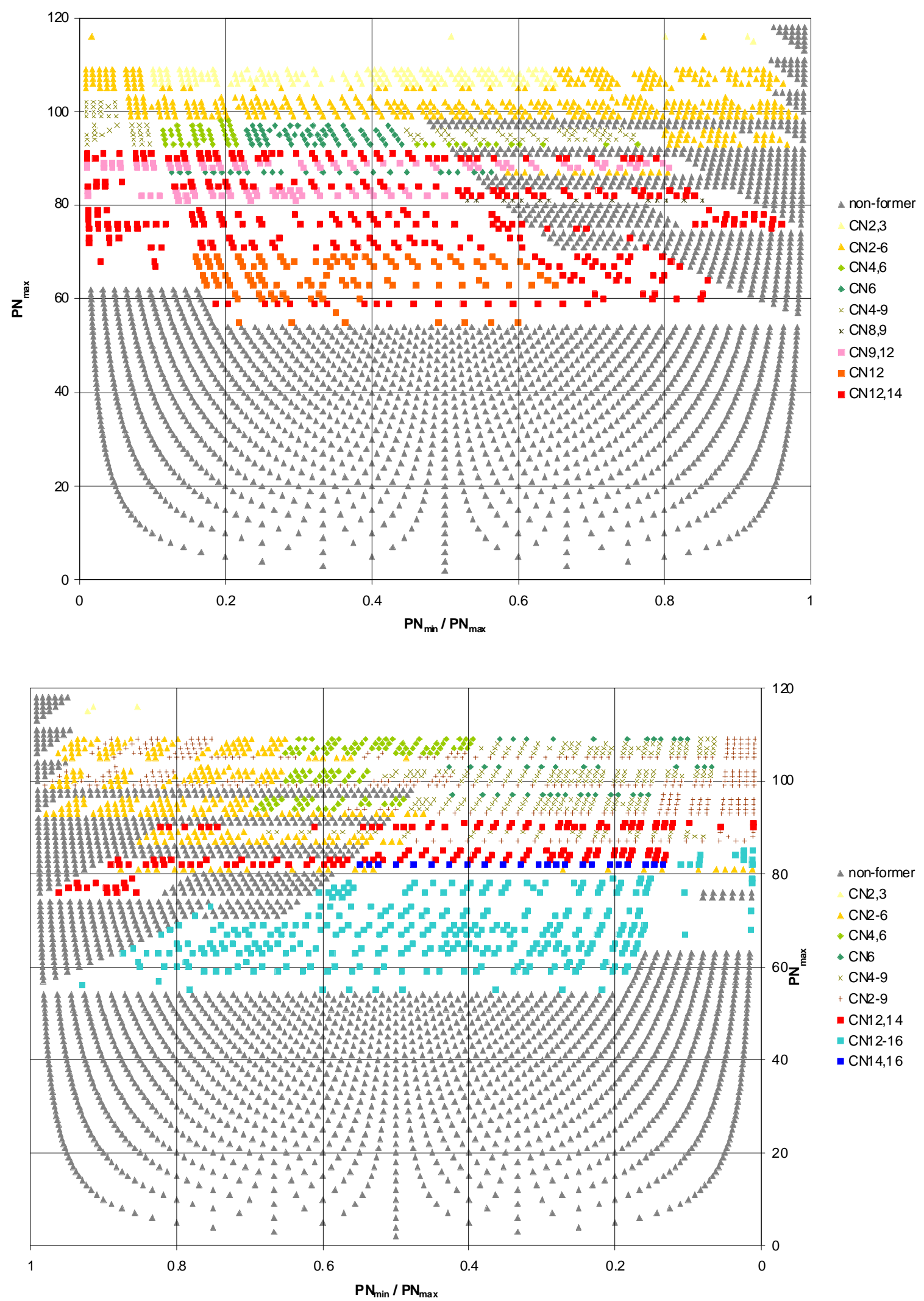

Fig. 6 Generalized AET stability map $P N_{M D \max }$ vs. $P N_{M D \min } / P N_{M D \max }$ which is independent of the stoichiometry and the number of chemical elements in the compound (based on 65,000 inorganic compounds, which have a [central atom-coordinating atoms] combination - AET occurrence count higher than 3). The upper map corresponds to $A-B$ combinations where the central atom $A$ has the highest Periodic Number $\left(P N_{M D \text { max }}\right)$, the bottom map to combinations where the central atom $A$ has the lowest Periodic Number $\left(P N_{M D \text { min }}\right)$. 
P. Villars et al., Data-driven generalized atomic environment prediction for binary and ...
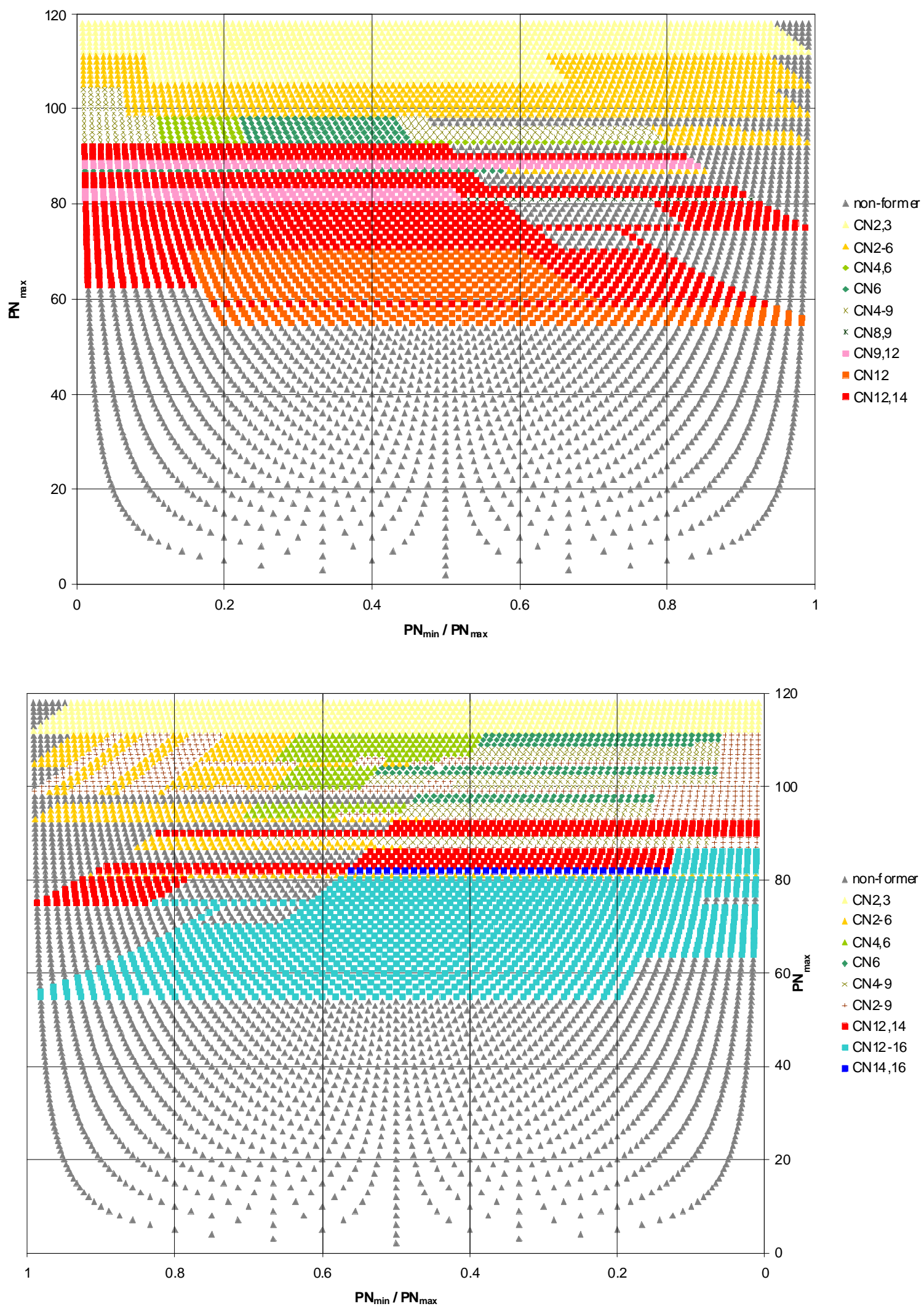

Fig. 7 Generalized AET stability map $P N_{M D \max }$ vs. $P N_{M D \min } / P N_{M D \max }$, which is independent of the stoichiometry and the number of chemical elements in an inorganic compound, prediction-completed for all possible [central atom-coordinating atoms] combinations. The upper map corresponds to $A-B$ combinations where the central atom $A$ has the highest Periodic Number $\left(P N_{M D \text { max }}\right)$, the lower map to combinations where the central atom $A$ has the lowest Periodic Number $\left(P N_{M D \text { min }}\right)$. 
Table 3 The 21 different Generalized Periodic Table - AET groups.

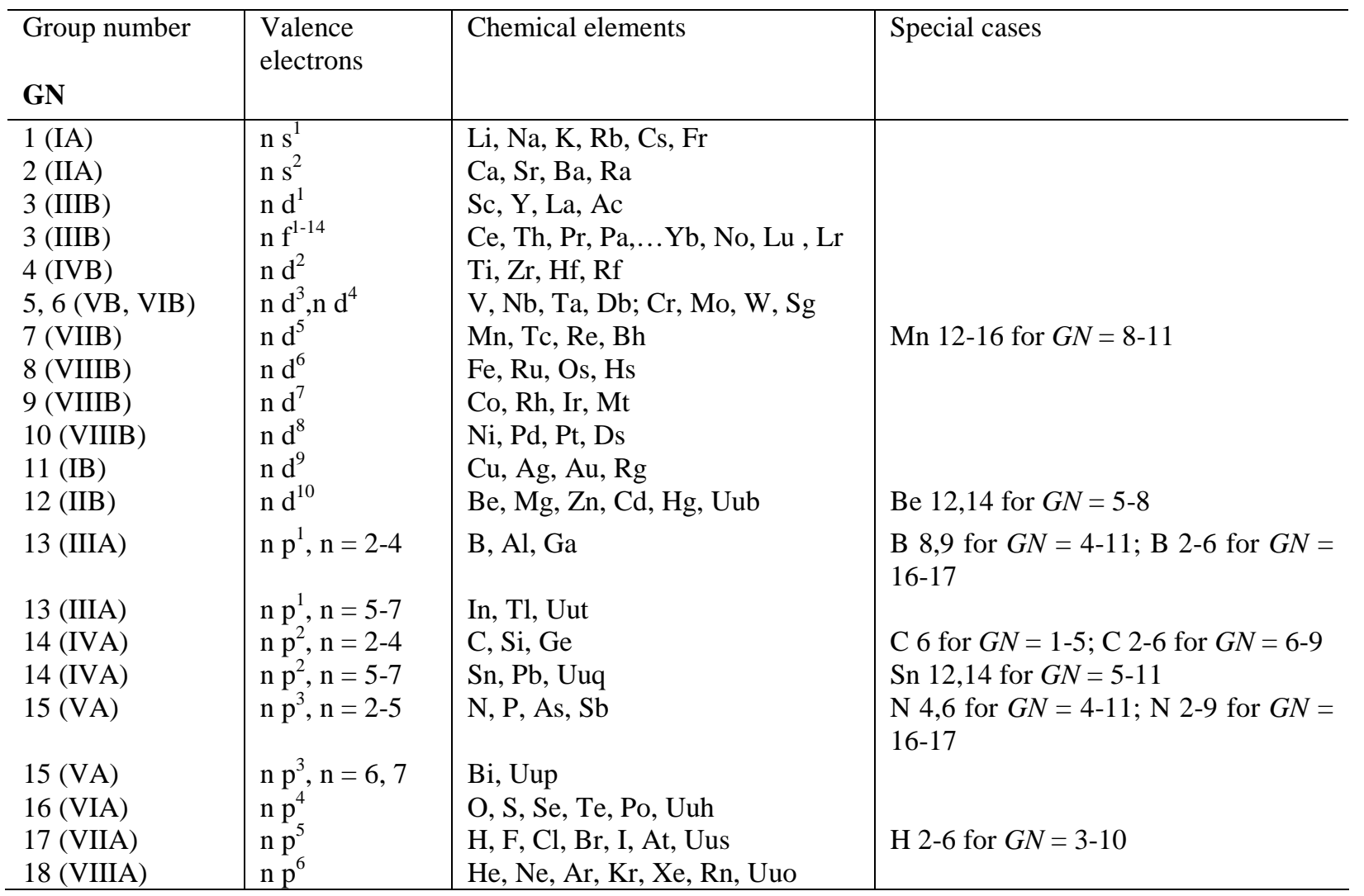

We may recall that "the Generalized AET matrix" and the "Generalized AET stability map" are not restricted to a specific stoichiometric ratio, nor to a particular number of chemical elements in the inorganic compound. Just one set of "Generalized AET matrix" or "AET stability map" is required to describe all inorganic compounds with respect of their most probable AETs.

\subsection{Periodic Table - Coordination Number (AET) graphs for the 18 different GNs}

A third way to present the same results is given by the fact that chemical elements with the same group number $G N$ are behaving in a similar way (except the $\mathrm{p}^{1}$ - to $\mathrm{p}^{3}$ - elements), and cannot be distinguished from each other. From Table 3 it can be seen that the dominant factor is the group number $G N$ (valence electron configuration), which is contained in the periodic number $P N_{M D}$, but not in the atomic number $A N$. The Periodic Table - Coordination Number (AET) graphs shown in Fig. 8 have the advantage that they are very easy to read. The reference chemical elements (shaded fields) represent the central atom $A$, the remaining chemical elements the coordinating atoms $B$. With the exception of systems involving $\mathrm{Mn}$, all binary systems involving chemical elements with the same group number $G N$ are non-formers. The 118 chemical elements can be separated into 21 major groups, which behave differently in context with the possible AETs, respectively the 'non-existence' of [central atom-coordinating atoms] combinations.

The atomic environments AETs for the elements acting as coordinating atoms $B$ located to the left of the column of the reference central atoms $A$ (e.g. $d^{7}$ elements $\mathrm{Co}, \mathrm{Rh}, \mathrm{Ir}, \mathrm{Mt})$ in the Periodic Table Coordination Number (AET) graphs are valenceelectron-governed by the central atom $A$. This is a consequence of the fact that the periodic number $P N_{M D}$ of these coordinating atoms $B$ is lower than the periodic number $P N_{M D}$ of the central atom $A$. In contrast, the atomic environments AETs formed by coordinating atoms $B$ located to the right of the column of the reference central atoms $A$ are valenceelectron-governed by the coordinating atoms $B$, because here $P N_{M D}(B)>P N_{M D}(A)$. Coordinating atoms $B$ within the same AET class domain (e.g. CN 4,6 ) can only have AETs corresponding to this class (tetrahedron, octahedron, pentagonal pyramid). For a central $\mathrm{Co}$ atom (or $\mathrm{Rh}, \mathrm{Ir}, \mathrm{Mt}$ ) this domain includes as possible coordinating atoms $\mathrm{F}, \mathrm{P}, \mathrm{S}, \mathrm{Cl}, \mathrm{As}, \mathrm{Se}, \mathrm{Br}$, $\mathrm{Sb}, \mathrm{Te}, \mathrm{I}, \mathrm{Po}, \mathrm{At}$, Uuh, and Uus. Each of these chemical elements can be either alone (e.g. $\mathrm{Cl}_{6}$ ), or in combination with up to two different ones as coordinating atoms (e.g. $\mathrm{Cl}_{4} \mathrm{BrSe}$ ) around the central atom. Chemical elements like $\mathrm{Be}, \mathrm{Mg}, \mathrm{Al}, \mathrm{Zn}, \mathrm{Ga}, \mathrm{Cd}$, $\mathrm{Hg}$, or Uub, which belong to the $\mathrm{CN} \mathrm{12,14}$ domain for the $d^{7}$-elements, cannot be mixed as coordinating atoms with chemical elements belonging to the above mentioned domain $\mathrm{CN} 4,6$. 
P. Villars et al., Data-driven generalized atomic environment prediction for binary and ...

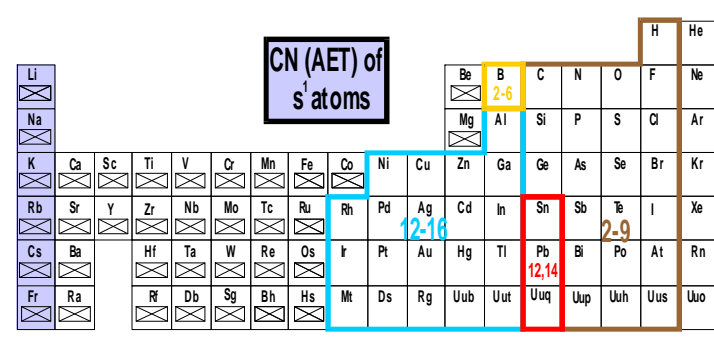

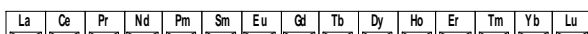

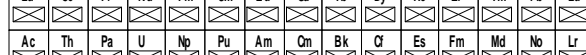

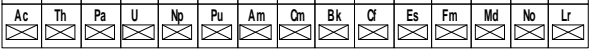

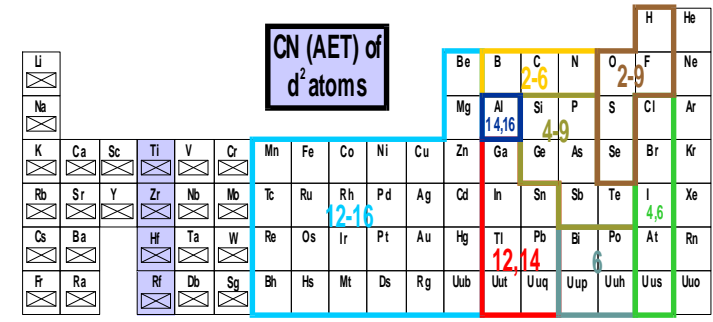

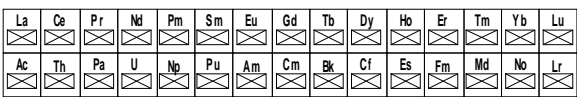
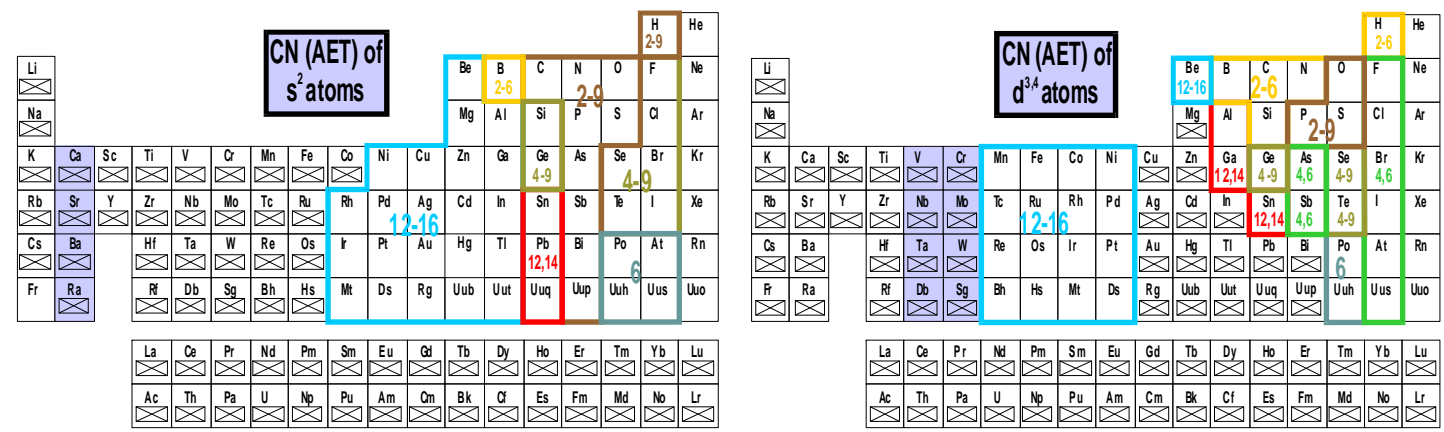

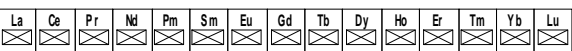
Ac

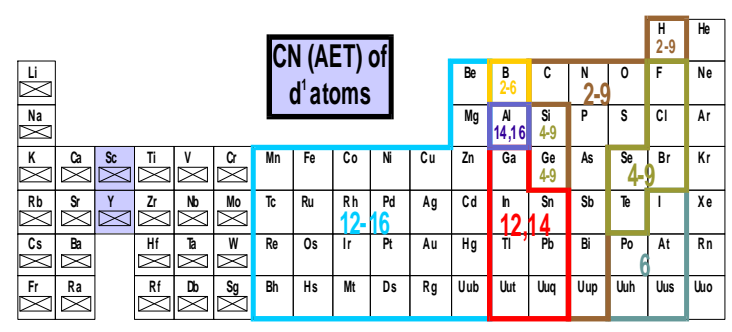

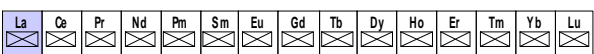

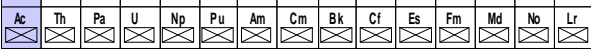

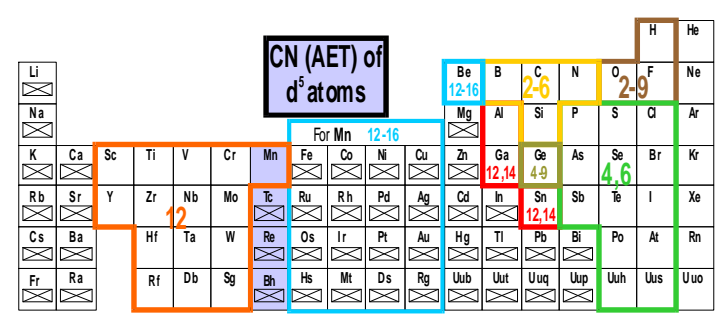

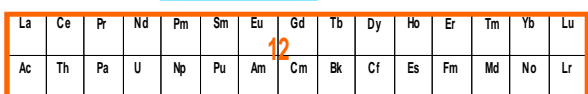
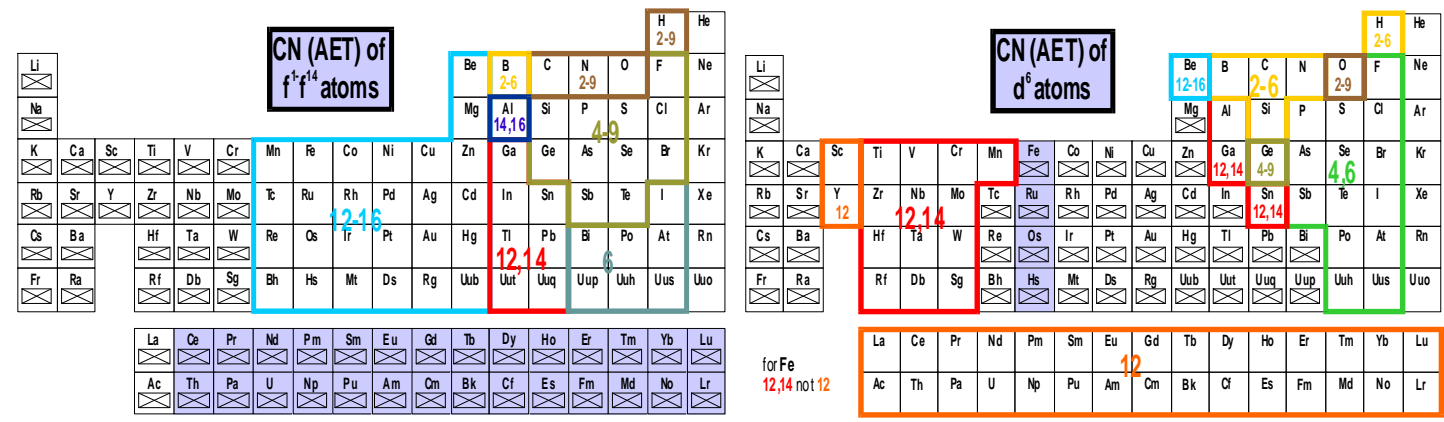

Fig. 8 The 21 Generalized Periodic Table - AET graphs for all possible [central atom-coordinating atoms] combinations, including not observed [central atom- coordinating atoms] combinations. Coordination numbers are defined for the former, the latter are marked by a 'cross in a box' symbol. The coordination numbers and their ranges are consistent with the AET classes defined in Table 2 (continued). 

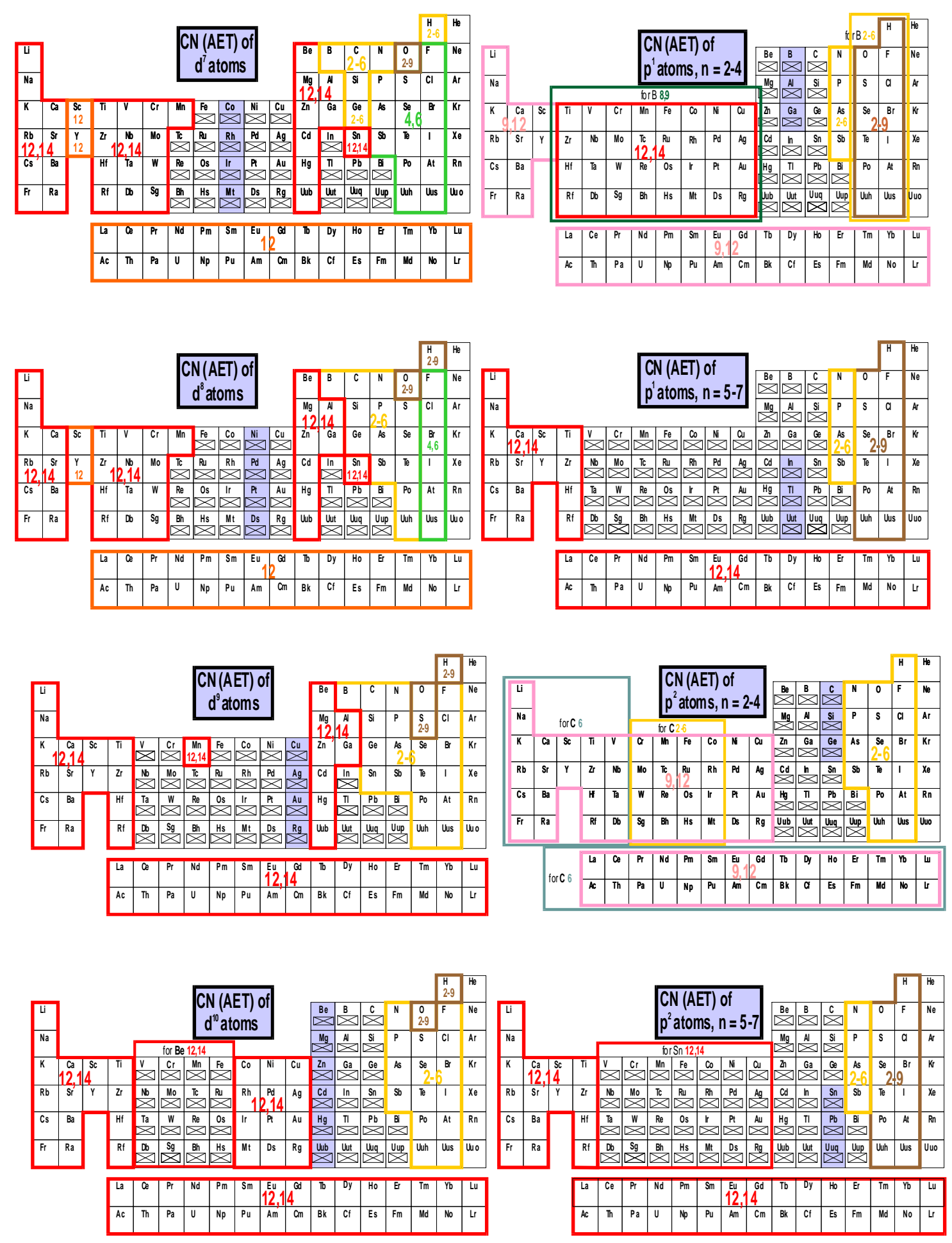

Fig. 8 The 21 Generalized Periodic Table - AET graphs for all possible [central atom-coordinating atoms] combinations, including not observed [central atom- coordinating atoms] combinations. Coordination numbers are defined for the former, the latter are marked by a 'cross in a box' symbol. The coordination numbers and their ranges are consistent with the AET classes defined in Table 2. (continued) 

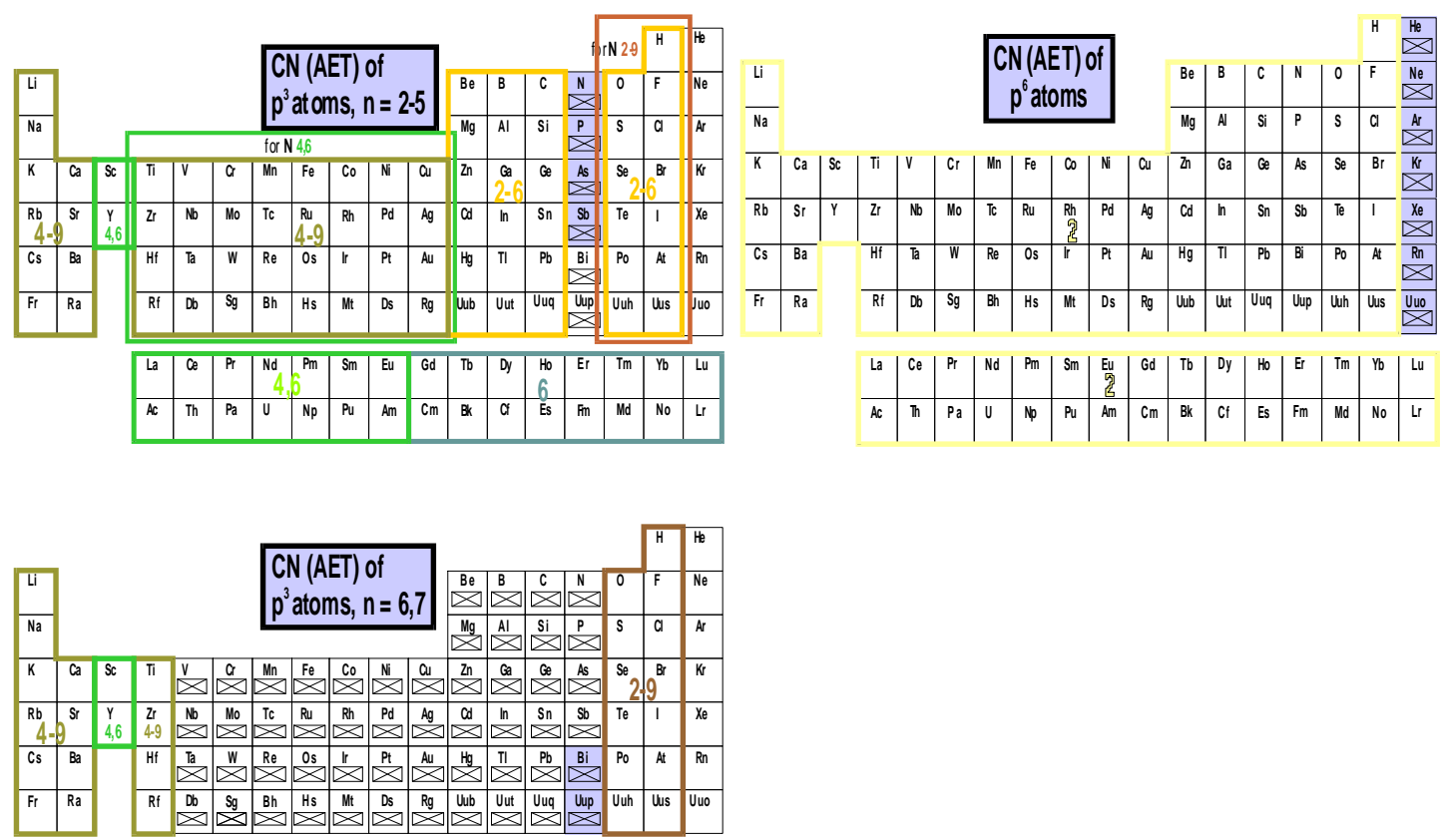

\begin{tabular}{|c|c|c|c|c|c|c|c|c|c|c|c|c|c|}
\hline La & $c_{0}$ & Pr & $N_{d}^{\text {dd }}$ & Sn & Еu & Go & Th & D & $\begin{array}{l}\text { Ho } \\
6 \\
6\end{array}$ & Er & \begin{tabular}{|l|l|} 
Tm \\
\end{tabular} & & Lu \\
\hline$A c$ & Th & $\mathrm{Pa}$ & \begin{tabular}{l|l} 
& $N$
\end{tabular} & $\mathrm{Pu}_{\mathrm{u}}$ & $\mathrm{Am}$ & $c_{n}$ & B & a & Es & Fin & Md & No & Lr \\
\hline
\end{tabular}
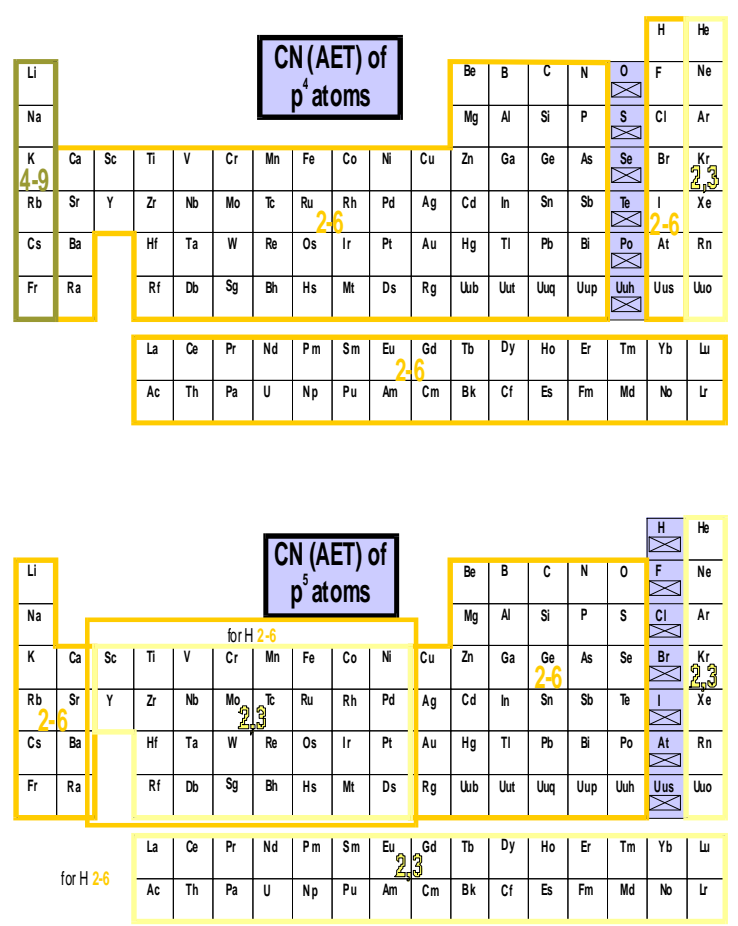

Fig. 8 The 21 Generalized Periodic Table - AET graphs for all possible [central atom-coordinating atoms] combinations, including not observed [central atom- coordinating atoms] combinations. Coordination numbers are defined for the former, the latter are marked by a 'cross in a box' symbol. The coordination numbers and their ranges are consistent with the AET classes defined in Table 2. 


\section{Conclusions}

Six observations in context with AETs, which are general and valid for the far majority of all inorganic compounds, are summarized below.

- The elements prefer a limited number of AETs such as non-collinear, non-coplanar triangle, tetrahedron, octahedron, pentagonal pyramid, icosahedron, cuboctahedron, and rhombic dodecahedron.

- The AETs are independent of the stoichiometry, as well as of the number of chemical elements in the inorganic compound; they depend only on the [central atom A - coordinating atoms B] combinations.

- There exist 21 different groups of chemical elements acting as central atom $A$, which prefer particular AETs for different coordinating elements $B$, or do not form [central atomcoordinating atoms] combinations with $B$.

- Chemical elements with $P N_{M D}>54$ define the AETs they are part of, regardless of whether they act as central or as coordinating atoms.

- The observation that the non-existence of [central atom-coordinating atoms] combinations is consistent with the non-existence of a binary equiatomic compound formed by the same elements demonstrates that if the 'reactivity difference' is too small in the equi-atomic binary case, it is also too small for [central atom-coordinating atoms] combinations within any AET, even when additional coordinating atoms would be included.

- AET class domains are clearly distinguished in the "Generalized AET matrix" as well as in the "Generalized AET stability map". The former is based on $\boldsymbol{P} N_{M D}(A)$ vs. $P N_{M D}(B)$, the latter on a combination of the periodic numbers $P N_{M D}$ of the constituent elements, $P N_{M D} \max$ vs. $P N_{M D \min } /$ $P N_{M D}$ max. It is now possible to predict the existence or 'non-existence' of [central atomcoordinating atoms] combinations in not yet studied systems and to assign probable AETs, or to search for inorganic compounds with a particular AET.

The present study proves that the analysis of critically evaluated data sets can lead to the discovery of so far "hidden" patterns, which can then be used to develop rules for semi-empirical materials design. To our own surprise it was possible in this work to develop a concentration-independent and number-ofchemical-element-independent generalized AET matrix and AET stability map. The 'payment' for it was that we had to group the 16 considered AETs in 12 AET classes, whereby we have in each class the choice between several AETs.

The generalized AET matrix, the generalized AET stability map and the Periodic Table - coordination number (AET) graphs can equally well be used for the most probable AET prediction and the results are identical. The preference for one of them is a matter of taste. Each representation highlights different aspects of the underlying pattern given through the AET data.

\section{Appendix}

\subsection{AET classification}

The atomic environment type is not a simple compound property in the sense that different atomic environment types are generally observed in the same inorganic compound. Within the data considered in this work the number of atomic environment types realized within one inorganic compound is as follows:

\begin{tabular}{|l|c|}
\hline 1 AET & $6 \%$ \\
\hline 2 different AETs & $29 \%$ \\
\hline 3 different AETs & $21 \%$ \\
\hline 4 different AETs & $11 \%$ \\
\hline 5 or more different AETs & $33 \%$ \\
\hline
\end{tabular}

In a single-environment type all atoms present in the structure have the same AET, in a two-environments type the atoms adopt two different AETs, and so on. It is also worth mentioning that structures with singleenvironment types are not limited to the simple chemical elements. On the other hand, twoenvironments types occur in element structures, as well as in binaries and multinaries. The same is valid for the three- and higher-environments types. In other words this means that in most inorganic compounds the same chemical element realizes two or more different AETs.

\subsection{Atomic Environment Types AETs}

The AETs are defined using the method of Brunner and Schwarzenbach [5], where all interatomic distances between the central atom and its coordinating atoms are plotted in a next-neighbor histogram. In most cases a clear maximum gap is defined. All atoms to the left of this maximum gap belong to the atomic environment type of the central atom. For those cases where no clear maximum gap is observed, a maximum-convex-volume rule is applied. In addition we keep the number of different AETs in a prototype as small as possible. It is obvious that a certain degree of ambiguity cannot be excluded, although we tried to be as consequent as possible. We like to point out that AET is a purely geometric concept and that, contrary to the coordination polyhedra as generally considered by chemists, cations and anions are not distinguished, and differences in atomic radii are not considered.

In order to determine the atomic environment types, as described in [4] and the references therein, complete crystal structure data must be available, i.e. space group, unit-cell dimensions, coordinates of the point-sets (atom sites) and their occupation. For the majority of the different prototypes adopted by inorganic compounds, however, the atomic environments are similar for isotypic compounds, so that the atom coordinates of the type-defining 
compounds can be used as a first approximation when only cell parameters are reported. Nevertheless, in this work only atomic environment type structures from fully refined structures were taken into consideration.

\subsection{Elemental-property Parameters}

Our recent work focusing on 39 elemental-property parameters [7] proved that these can be subdivided into the following five distinct groups:

- atomic number $A N$ (e.g. main quantum number, atomic mass)

- periodic number $P N$ (e.g. group number, valence electron number)

- size SZ (e.g. Zunger's pseudo-potential radii, Pauling's covalent radii)

- reactivity $R E$ (e.g. Pauling's electronegativity, Martinov-Batsanov's electronegativity)

- cohesion energy $C E$ (e.g. enthalpy of formation, melting temperature)

The atomic number $A N$ of the elements together with their periodic number $P N$ were found to form an efficient pair for the discussion of metallurgical and structural problems. The periodic number $P N$ represents a different enumeration of the elements, emphasizing the role of the valence electrons. In contrast to the atomic number, $P N$ depends in details on the underlying Periodic Table of the elements. The elemental-property parameters 'atomic size $S Z_{a}$ ' and 'atomic reactivity $R E_{a}$ ' can be derived from fits of various experimental and theoretical data sets. These two parameters can be approximated as simple functions of $A N$ and $P N$ :

$S Z_{\mathrm{a}}=\mathrm{k}_{\mathrm{SZ}}[\log (A N+1)]\left[\mathrm{k}_{\mathrm{PN}}-(\log P N)^{3}\right]$

$R E_{\mathrm{a}}=\mathrm{k}_{\mathrm{RE}}\left\{[\log (A N+1)]\left[\mathrm{k}_{\mathrm{PN}}-(\log P N)^{3}\right]\right\}-1=$ $\mathrm{k}_{\mathrm{SZ}} \mathrm{k}_{\mathrm{RE}}\left(S Z_{a}\right)-1$

where $k_{\mathrm{PN}}$ is a scaling factor, and $\mathrm{k}_{\mathrm{SZ}}, \mathrm{k}_{\mathrm{RE}}$ are fit parameters for a fit to experimental data. We argue that all elemental-property parameter patterns are derived from $A N$ and $P N$. $A N$ and $P N$ represent fundamental elemental-property parameters independent of each other. Any pattern, which shows well-defined functional behavior within each group number $G N$, as well as within each main quantum number $Q N$, can be considered.

\section{Acknowledgements}

The authors are grateful to Dr. F. Hulliger for his interest in the work and for his critical comments during the careful reading of the manuscript.

\section{References}

[1] P. Villars, K. Brandenburg, M. Berndt, S. LeClair, A. Jackson, Y.-H. Pao, B. Igelnik, M. Oxley, B. Bakshi, P. Chen, S. Iwata, J. Alloys Compd. 317-318 (2001) 26-38.

[2] P. Villars, K. Cenzual, J. Daams, Y. Chen, S. Iwata, J. Alloys Compd. 367 (2004) 167-175.

[3] P. Villars, K. Cenzual (Eds.), Crystal Structures of Inorganic Compounds, Landolt-Börnstein, New Series, Springer-Verlag, Berlin, 2004-8, Vol. III/43A, pp. IX-XIII.

[4] J.L.C. Daams, in: J.H. Westbrook, R.L. Fleischer (Eds.), Intermetallic Compounds, John Wiley and Sons, New York, 1994, Vol. 1, pp. 363-383.

[5] G.O. Brunner, D. Schwarzenbach, Z. Kristallogr. 133 (1971) 127-133.

[6] P. Villars, K. Cenzual (Eds.), Pearson's Crystal Data CD-ROM, release 2007/8, ASM International, Materials Park, Ohio, 2007.

[7] P. Villars, J. Daams, Y. Shikata, K. Rajan, S. Iwata, Chem. Met. Alloys 1 (2008) 1-23.

[8] D.G. Pettifor, Solid State Commun. 51 (1984) 31.

[9] H. Okamoto, Binary Alloy Phase Diagrams, Desk Edition, ASM International, Materials Park, Ohio, 2001.

[10] P.Villars, M. Berndt, K. Brandenburg, K. Cenzual, J. Daams, F. Hulliger, T.Massalski, H. Okamoto, K. Osaki, A. Prince, H. Putz, S. Iwata (Eds.), PAULING FILE, Binaries Edition, ASM International, Materials Park (Ohio), 2002.

[11] P. Villars, H. Okamoto, K. Cenzual (Eds.), ASM Alloy Phase Diagrams Center, online release 2007/8, ASM International, Materials Park, Ohio, 2007.

[12] F.R. de Boer, R. Boom, W.C.M. Mattens, A.R. Miedema, A.K. Niessen, in: F.R. de Boer, D.G. Pettifor (Eds.), Cohesion in Metals, NorthHolland, Amsterdam, 1988, pp. 13-94. 